\title{
Body Parts Matter: Social, Behavioral, and Biological Considerations for Urethral, Pharyngeal, and Rectal Gonorrhea and Chlamydia Screening Among MSM in Lima, Peru
}

Passaro, R. Colby MPH ${ }^{1,2}$; Segura, Eddy R. MD, MPH ${ }^{2,3}$; Perez-Brumer, Amaya MSc${ }^{4}$; Cabeza, Jeanne MD, MPH ${ }^{2}$; Montano, Silvia M. MD, MPH ${ }^{5}$; Lake, Jordan E. MD, MSc ${ }^{2,6}$; Sanchez, Jorge MD, MPH ${ }^{7,8,9}$; Lama, Javier R. MD, MPH ${ }^{7,9}$; Clark, Jesse L. MD, MSc ${ }^{2}$

Sexually Transmitted Diseases: February 20, 2018 - Volume Publish Ahead of Print - Issue $\mathrm{p}$

doi: 10.1097/OLQ.0000000000000816

Original Study: PDF Only

1 College of Medicine, University of Tennessee Health Science Center, Memphis, TN, USA

2 South American Program in HIV Prevention Research, David Geffen School of Medicine, University of California Los Angeles, Los Angeles, CA, USA

3 Escuela de Medicina, Universidad Peruana de Ciencias Aplicadas, Lima, Peru

4 Columbia University Mailman School of Public Health, New York, NY, USA

5 US Naval Medical Research Unit-6, Callao, Peru

6 McGovern Medical School at UTHealth, Department of Internal Medicine, Division of Infectious Diseases, Houston, TX, USA

7 Asociación Civil Impacta Salud y Educación, Lima, Peru

8 Centro de Investigaciones Tecnológicas Biomédicas y Medioambientales, Lima, Peru

9 Department of Global Health, University of Washington, Seattle, WA, USA

Corresponding author: R. Colby Passaro, MPH, ryan.c.passaro@vanderbilt.edu. PH: 310601-7798, FAX: 713-500-5495

We have no conflicts of interest to disclose.

\section{Abstract}

Background Gonorrhea (GC) and chlamydia (CT) disproportionately affect men who have sex with men (MSM), and public health implications vary by anatomic site and bacterial agent. Urethral and rectal GC and CT can increase risk of HIV transmission, while pharyngeal GC may be a reservoir for antimicrobial resistance. To define screening priorities in Latin America, we compare differences in the prevalence and correlates of urethral, pharyngeal, and rectal GC and CT among MSM in Peru.

Methods A cross-sectional sample of 787 MSM from Lima was screened between 2012-2014. We described prevalence of urethral, pharyngeal, and rectal GC and CT infection and conducted bivariate analyses of associations with social, behavioral, and biological characteristics. Poisson regression analyses assessed the correlates of each infection at each anatomic site.

Results The most commonly symptomatic infection (urethral GC; $42.1 \%$ ) was the least prevalent (2.4\%). The most prevalent infections were rectal CT (15.8\%) and pharyngeal GC (9.9\%). Rectal CT was the least commonly symptomatic (2.4\%) infection, and was associated with younger age (aPR, $95 \% \mathrm{Cl}: 0.96,0.94-0.98)$, HIV infection (1.46, 1.06-2.02), and pasivo (receptive; 3.59, 1.62-7.95) and moderno (versatile; $2.63,1.23-5.60)$ sexual roles. 
Conclusions Results highlight limitations of current syndromic screening strategies for STDs in Peru, wherein urethral CT and rectal GC and CT may be missed due to their frequently asymptomatic presentations. Successful management of GC and CT infections among MSM in low-resource settings requires differentiating between bacterial agent, symptomatic presentation, associated risk factors, and public health implications of untreated infection at different anatomic sites.

SOURCES OF SUPPORT: Funding for this work was provided by the US National Institute of Health grants NIH R25 MH087222 to JLC, R21 MH 092322, and K23 Al110532 to JEL

SHORT SUMMARY: High prevalence of asymptomatic rectal chlamydia and pharyngeal gonorrhea among MSM in Lima, Peru; rectal CT was most common and associated with younger age, HIV infection, receptive sexual role.

(C) Copyright 2018 American Sexually Transmitted Diseases Association 